\title{
Resolving distant, dusty galaxies using observations and simulations
}

\author{
R. K. Cochrane ${ }^{\mathbb{1}}$ \\ SUPA, Institute for Astronomy, Royal Observatory Edinburgh, EH9 3HJ, UK
}

\begin{abstract}
Spatially resolved studies of galaxies in the high-redshift Universe have traditionally been reliant on data at rest-frame optical and UV wavelengths, which can be biased towards the least dust-obscured galaxies. For several years now, we have been able to resolve and probe the morphology of longer-wavelength emission from distant galaxies with ALMA, and a number of recent ALMA studies were presented at the IAU Symposium No. 352. These included our study of the resolved multi-wavelength emission of galaxies at $z \sim 2$. As part of the SHiZELS collaboration, we are mapping the $\mathrm{H} \alpha$ emission line (from SINFONI/VLT), UV continuum (from HST), and the far-infrared (from ALMA) emission from a small sample of $\mathrm{H} \alpha$-selected galaxies. In this proceedings paper, we showcase the high quality of our data, and the spectacular structures displayed by one of our most dusty sources. We also provide an overview of some highly complementary simulation-based work, using galaxies drawn from the FIRE-2 zoom-in cosmological hydrodynamical simulations. Using sophisticated radiative transfer techniques, we have derived predictions for the spatially-resolved emission of a sample of star-forming galaxies, from rest-frame far-ultraviolet to the far-infrared. For both observed and simulated galaxies, emission maps show striking differences with wavelength, with the same galaxy appearing clumpy and extended in the far-ultraviolet yet compact at far-infrared wavelengths.
\end{abstract}

Keywords. galaxies: high-redshift, galaxies: starburst, infrared: galaxies, radiative transfer

\section{Introduction}

Recent far-infrared (FIR) observations have shown that $\sim 85 \%$ of the total star formation at $z \sim 2$ is enshrouded in dust (Dunlop et al. 2017). For high-mass galaxies $\left(M_{*}>2 \times 10^{10} M_{\odot}\right)$, the SFR derived from long-wavelength FIR emission is an extraordinary 200 times that derived from unobscured light. It is therefore crucial to study the internal properties of massive $z \sim 2$ galaxies at long wavelengths, where dust emits thermally, as well as at shorter wavelengths. In this paper, we present two complementary approaches. The first involves an ambitious observational programme in which we compare several widely-used tracers of star-formation for an individual high-redshift source. The second couples high-resolution zoom-in simulations with radiative transfer to understand the physics at play as well as the observational biases that affect what is measured.

\section{Mapping $\mathrm{z} \sim 2$ galaxies at multiple wavelengths}

The High-Redshift(Z) Emission Line Survey, HiZELS, used a combination of narrowband and broad-band filters to select thousands of intermediate redshift star-forming galaxies via their emission line fluxes (Sobral et al. 2013, 2015) in fields with high-quality multi-wavelength coverage (COSMOS, UDS \& SA22). Following a number of studies of the global properties of these galaxies (e.g. Sobral et al. 2009, 2010, 2014; Cochrane et al. 2017 , 2018), the HiZELS collaboration has recently obtained resolved maps of a sample of 


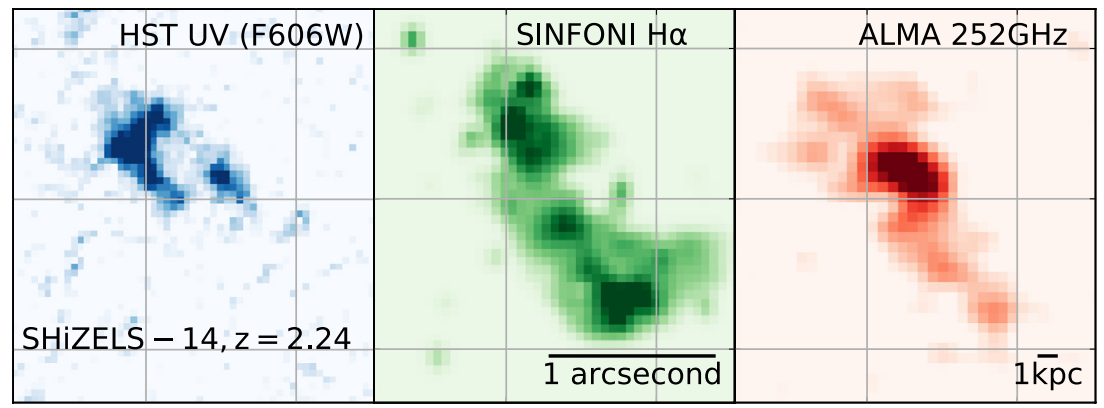

Figure 1. SHiZELS-14, a star-forming galaxy at $z=2.2$, mapped at matched $\sim 1 \mathrm{kpc}$ resolution at three wavelengths typically used to probe star formation: rest-frame UV (HST F606W filter), the $\mathrm{H} \alpha$ emission line (SINFONI/VLT), FIR dust continuum emission (ALMA). The three images show strikingly different morphologies, with little short-wavelength emission from the most dusty central region. The distribution of star formation inferred from each of the tracers is distinct. From Cochrane et al. (in prep).

$\mathrm{H} \alpha$-selected galaxies at $z \sim 1.5$ and $z \sim 2.2$. We have obtained $\sim 0.15^{\prime \prime}$ matched-resolution observations of $\mathrm{H} \alpha$ emission (using adaptive optics with the SINFONI Integral Field Unit on the VLT; see Swinbank et al. 2012a,b; Molina et al. 2017), dust continuum emission (with ALMA), and in the rest-frame UV and optical (from HST UVIS imaging).

One of our most extensively imaged galaxies is SHiZELS-14 (Figure 1), a $M_{*} \sim 10^{11} M_{\odot}$, highly star-forming galaxy at $z=2.24$. Although identified via its $\mathrm{H} \alpha$ emission, this galaxy displays a bright sub-millimeter flux density $\left(S_{\mathrm{obs}}, 252 \mathrm{GHz}=2.7 \pm 0.15 \mathrm{mJy}\right)$, and is not a typical star-forming galaxy, as we might have have inferred from its modest UV and $\mathrm{H} \alpha$ fluxes. Fitting the dust SED with a two grey-body model yields a dust mass of $M_{\text {dust }}=10^{9.1 \pm 0.1} M_{\odot}$, a TIR luminosity of $\log _{10}\left(L_{\mathrm{TIR}} / \mathrm{erg} \mathrm{s}^{-1}\right)=46.39 \pm 0.02$ and a star formation rate of $\mathrm{SFR}=950 \pm 50 \mathrm{M}_{\odot} \mathrm{yr}^{-1}$. This bright IR emission places it in the category of a ULIRG. The star formation rates derived from shorter wavelength data using standard luminosity-SFR calibrations (Kennicutt \& Evans 2012) are notably lower $\left(\mathrm{SFR} \sim 10-200 \mathrm{M}_{\odot} \mathrm{yr}^{-1}\right)$, highlighting the importance of long-wavelength data for such dusty systems.

Figure 1 shows the impressive quality of our $\sim 0.15^{\prime \prime}$ data, and highlights the biased view of galaxy morphology provided by UV data. After careful astrometric calibration, the UV emission is spatially offset from the dust emission, and does not represent the position of the bulk of star formation. A number of recent studies have found similar offsets in the multi-wavelength emission of bright sub-millimeter galaxies (e.g. Hodge et al. 2016; Chen et al. 2017; Calistro Rivera et al. 2018).

\section{Simulating multi-wavelength emission with FIRE-2}

In this section, we describe work undertaken to simulate the emission from galaxies at high spatial resolution. The Feedback In Realistic Environments (FIRE) project (Hopkins et al. 2014, 2018) is a set of hydrodynamical cosmological zoom-in simulations that explore the role of stellar feedback in galaxy formation and evolution. FIRE simulations explicitly model multi-channel stellar feedback by resolving the formation of giant molecular clouds and also include models for both energy and momentum return from the main stellar feedback processes, using predictions of stellar population synthesis models without the extensive parameter tuning employed in other simulations. They broadly reproduce many observed galaxy properties, including stellar masses, star-formation histories and the 'main sequence' of star-forming galaxies (see Hopkins et al. 2014; Sparre 
et al. 2017), metallicities and abundance ratios (Ma et al. 2016; van de Voort et al. 2015), as well as morphologies and kinematics of both thin and thick disks (Ma et al. 2017).

Here, we summarise our study of the central galaxies of four massive haloes recently published by Cochrane et al. (2019). These haloes were originally selected and simulated by Feldmann et al. (2016, 2017) with the original FIRE model (Hopkins et al. 2014) as part of the MAssiveFIRE suite. Our sample of central galaxies have dark matter halo masses of $M_{\text {halo }} \sim 10^{12.5} M_{\odot}$ and stellar masses of $7 \times 10^{10}-3 \times 10^{11} M_{\odot}$ at $z=2$. The mass resolution of our simulations is $3.3 \times 10^{4} M_{\odot}$ for gas and star particles and $1.7 \times 10^{5} M_{\odot}$ for dark matter.

To simulate the multi-wavelength emission from these galaxies in a consistent way, we perform radiative transfer. We model the radiation field from stars as a flow of photons through the dusty medium of a galaxy to compute the effects of dust absorption, scattering, and re-emission of the absorbed light, including dust self-absorption. We implement this using the Stellar Kinematics Including Radiative Transfer (SKIRT) Monte Carlo radiative transfer code (Baes et al. 2011; Camps \& Baes 2015). We extract gas and star particles from the FIRE-2 simulations at a number of time snapshots. For gas particles with temperature $<10^{6} \mathrm{~K}$, we compute dust masses using the metallicity of the gas particles and a dust-to-metals mass ratio of 0.4 (Dwek 1998; James et al. 2002). We assume that dust is destroyed in gas particles with temperature $>10^{6} \mathrm{~K}$ (Draine \& Salpeter 1979; Tielens et al. 1994). We use a Weingartner \& Draine (2001) Milky Way dust prescription to model a mixture of graphite, silicate and PAH grains. Star particles are assigned Bruzual \& Charlot (2003) SEDs according to their ages and metallicities. We model the flux that would be received by an observer on Earth at 100 discrete wavelengths between rest-frame UV and FIR, also considering different inclinations with respect to the disk plane of the galaxy.

Figure 2 shows an example of the output of our radiative transfer for a simulated galaxy at $z=2.95$. The derived morphologies are notably different in different wavebands, with the same galaxy appearing clumpy in the far-ultraviolet yet regular and spiral-like at farinfrared wavelengths. The observed-frame $870 \mu \mathrm{m}$ half-light radii of our FIRE-2 galaxies are $\sim 0.5-4 \mathrm{kpc}$, consistent with existing ALMA observations of high redshift galaxies. In these simulated galaxies, the dust continuum emission is more compact than the cold gas, but more extended than the stellar component. The most extreme cases of compact dust emission are driven by particularly compact recent star formation, which can drive steep dust temperature gradients.

\section{Summary}

In this short paper, we have provided an overview of our ongoing observational and simulation-based work to understand the spatially-resolved, multi-wavelength emission from highly star-forming galaxies around the peak of cosmic star formation. As well as shaping the global SED, dust clearly plays a role in determining the spatial extent of emission observed in different wavebands. For dusty galaxies like SHiZELS-14, short wavelength emission appears to trace little but holes in the dust distribution. Our radiative transfer modelling confirms that dust obscuration can drive substantial decoupling in the spatial distributions of short and long-wavelength light. For our simulated dusty galaxies, the UV is a poor tracer of not only the amount of star formation but also its spatial extent.

\section{Acknowledgements}

Section 2 is based on work conducted in collaboration with the SHiZELS team, including Philip Best, Mark Swinbank, Edo Ibar, Ian Smail, David Sobral, and Juan 


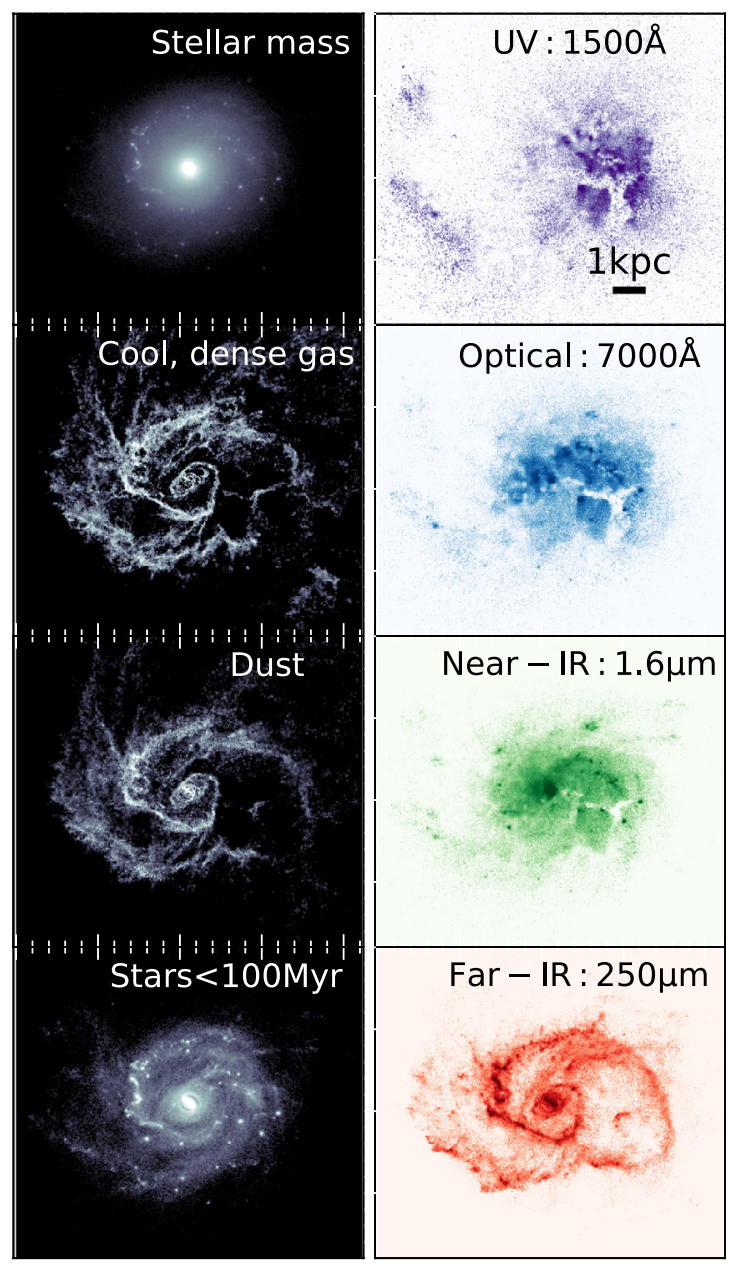

Figure 2. The wavelength-dependent morphology of a $M_{*} \sim 10^{11}$ galaxy in the FIRE-2 simulations at $z=2.95$. The left-hand panels show the projected distributions of stellar mass, cold, dense gas mass, dust mass, and stars formed within 100Myr (intrinsic properties of the galaxy). The right-hand panels show the SKIRT-predicted images at different rest-frame wavelengths. The morphology is strongly dependent on the wavelength. The galaxy appears clumpy and extended in the rest-frame UV but more ordered at longer wavelengths. The UV and optical light is significantly offset from the peak of the stellar mass and SFR, appearing to trace holes in the dust. The colour scales are logarithmic and span the $70^{\text {th }}-99^{\text {th }}$ percentiles of the flux distribution of each panel, to highlight the qualitative differences in morphology. From Cochrane et al. (2019).

Molina. This project will be published in a forthcoming paper. Section 3 is based on work with FIRE collaborators, Christopher Hayward, Daniel Anglés-Alcázar, Jennifer Lotz, Tyler Parsotan, Xiangcheng Ma, Dusan Kereš, Robert Feldmann, Claude-André Faucher-Giguère, and Philip Hopkins. This work was published in Cochrane et al. (2019).

\section{References}

Baes, M. et al. 2011, ApJS, 196, 22

Bruzual, G. \& Charlot, S. 2003, MNRAS, 35, 1

Calistro Rivera, G. et al. 2018, ApJ, 863, 56

Camps, P. \& Baes, M. 2015, Astron. Comput., 9, 20 
Chen, C.-C. et al. 2017, ApJ, 846, 108

Cochrane, R. K., Best, P. N., Sobral, D., Smail, I., Wake, D. A., Stott, J. P., \& Geach, E. 2017, MNRAS, 469, 2913

Cochrane, R. K., Best, P. N., Sobral, D., Smail, I., Geach, J. E., Stott, J. P., \& Wake, D. A. 2018, MNRAS, 475, 3730

Cochrane, R. K. et al. 2019, MNRAS, 488, 1779-1789

Draine, B. T. \& Salpeter, E. E. 1979, ApJ, 231, 77

Dunlop, J. S. et al. 2017, MNRAS, 466, 861

Dwek, E. 1998, ApJ, 501, 643

Feldmann, R., Hopkins, P. F., Quataert, E., Faucher-Giguère, C.-A., \& Kereš, D. 2016, MNRAS, 458, L14

Feldmann, R., Quataert, E., Hopkins, P. F., Faucher-Giguère, C. A., \& Kereš, D. 2017, MNRAS, 470,1050

Kennicutt, R. C. \& Evans, N. J. 2012, ARA\&广A, 50, 531

Hodge, J. A. et al. 2016, ApJ, 833, 103

Hopkins, P. F., Kereš, D., Oñorbe, J., Faucher-Giguère, C. A., Quataert, E., Murray, N., \& Bullock, J. S. 2014, MNRAS, 445, 581

Hopkins, P. F. et al. 2018, MNRAS, 480, 800

James, A., Dunne, L., Eales, S., \& Edmunds, M. G. 2002, MNRAS, 335, 753

Ma, X., Hopkins, P. F., Faucher-Giguère, C. A., Zolman, N., Muratov, A. L., Kereš, D., \& Quataert, E. 2016, MNRAS, 456, 2140

Ma, X., Hopkins, P. F., Wetzel, A. R., Kirby, E. N., Anglés-Alcázar, D., Faucher-Giguère, C.-A., Kereš, D., \& Quataert, E. 2017, MNRAS, 467, 2430

Molina, J., Ibar, E., Swinbank, A. M., Sobral, D., Best, P. N., Smail, I., Escala, A., \& Cirasuolo, M. 2017, MNRAS, 466, 892

Sobral, D. et al. 2009, MNRAS, 398, 75

Sobral, D., Best, P. N., Geach, J. E., Smail, I., Cirasuolo, M., Garn, T., Dalton, G. B., \& Kurk, J. 2010, MNRAS, 1563, 1551

Sobral, D. et al. 2013, ApJ, 779, 139

Sobral, D., Best, P. N., Smail, I., Mobasher B., Stott J., \& Nisbet D. 2014, MNRAS, 427, 3516

Sobral, D. et al. 2015, MNRAS, 451, 2303

Sparre, M., Hayward, C. C., Feldmann, R., Faucher-Giguère, C. A., Muratov, A. L., Kereš, D., \& Hopkins, P. F. 2017, MNRAS, 466, 88

Swinbank, A. M., Sobral, D., Smail, I., Geach, J. E., Best, P. N., Mccarthy, I. G.,Crain, R. A., \& Theuns, T. 2012a, MNRAS, 426, 935

Swinbank, A. M., Smail, I., Sobral, D., Theuns, T., Best, P. N., \& Geach, J. E. 2012b, ApJ, 760,13

Tielens, A. G. G. M., McKee, C. F., Seab, C. G., \& Hollenbach, D. J. 1994, ApJ, 431, 321

Weingartner, J. C. \& Draine, B. T. 2001, ApJ, 548, 296

van de Voort, F., Quataert, E., Hopkins, P. F., Kereš, D., Faucher-Giguère, C. A. 2015, MNRAS, 447,140 